\title{
Multidisciplinary Approach for a Patient with Severely Worn Dentition by Full Mouth Rehabilitation-Restoration of Esthetics, Function and Oral Health: A Clinical Case Report
}

\author{
Shilpa Sinnurkar \\ Assistant Professor, Department of Prosthodontics Coorg Institute of Dental Sciences, Virajpet-571218 Karnataka India
}

\begin{abstract}
The management of tooth wear, especially attrition has become a subject of interest in the Prosthodontic literature, both from a preventive and a restorative point of view. The rationales for altering vertical dimension include aesthetic, correcting occlusal relationship, and allowing space for restorations for prosthetic convenience. A critical aspect for a severely attrited teeth by full mouth rehabilitation is to determine the occlusal vertical dimension (OVD) and the inter-occlusal rest space (IRS). There is great apprehension involved in reconstructing debilitated dentition due to widely divergent views concerning the choice of an appropriate occlusal scheme for successful full mouth rehabilitation. A systematic approach for managing tooth wear can lead to a predictable and favorable prognosis. This case report highlights the stages for rehabilitation, from diagnosis to final treatment and follow up for a patient with severe tooth wear. The full mouthrehabilitation procedure includes increasing the vertical dimension according to Pan-key-Mann Schuyler (PMS) philosophy followed by restoration of missing and attrited teeth with metal ceramic restorations and a removable cast partial denture (CPD).
\end{abstract}

Keywords: Attrition, Vertical dimension of occlusion (VDO), Inter-occlusal rest space (IRS), Occlusal Splints, Full Mouth Rehabilitation

\section{Introduction}

The term 'tooth wear' is a general term that can be used to describe the surface loss of dental hard tissues from causes other than dental caries, trauma or as a result of developmental disorders. It is a normal physiological process that is macroscopic, irreversible and is cumulative with age [1, 2]. Lambrechts et al. in 19892 estimated the normal vertical loss of enamel from physiological wear to be approximately 20-38 $\mu \mathrm{m}$ per annum [3]. Tooth wear can be classified as attrition, abrasion, and erosion depending on its cause. A differential diagnosis is not always possible because, in many situations, there exists a combination of these processes. However, clinically it is difficult to isolate a single etiological factor when a patient presents with tooth wear. For the latter reason, the term 'tooth surface loss' (TSL) was suggested by Eccles in 19823 to embrace all of the etiological factors regardless of whether the exact cause of wear has been identified[4]. This includes factors such as trauma, developmental conditions such as Amelogenesis and Dentinogenesis Imperfecta, and Iatrogenic wear [5].

Therefore, it is utmost essential to identify the factors that contribute to excessive wear and to evaluate alteration of the VDO caused by the worn dentition. In many cases, the vertical dimension of occlusion (VDO) is maintained by tooth eruption and alveolar bone growth. As teeth are worn, the alveolar bone undergoes an adaptive process and compensates for the loss of tooth structure to maintain the VDO [6]. Therefore, VDO should be conservative and should not be changed without careful approach. Especially, increasing the VDO in bruxers puts a severe overload on the teeth and often results in the destruction of the restorations or teeth themselves [7].
In daily clinical practice, patients request prosthetic rehabilitation to restore unstable occlusion resulting from extensive tooth wear and early loss of permanent teeth. However, a limited inter-occlusal space creates a challenge for restorative and prosthetic treatment because the space required for restoration is unavailable, and it is probable that the retention and resistance form will be inadequate for the final prosthesis. The objective of full mouth rehabilitation is not only the reconstruction and restoration of the worn out dentition, but also maintenance of the health of the entire stomatognathic system. Restorative dentists have always sought to understand, capture, and recreate the movements of the mandible in an attempt to create a mechanical simulation of the patient upon which prostheses can be fabricated. Successful re-creation of each individual patient's unique functional and non-functional movements would greatly improve the final prosthetic device when delivered to the patient.

\section{Case Description}

Rehabilitation of the chewing function and acceptable aesthetics were the major treatment goals. Nevertheless, it was difficult to develop a treatment plan that met the patient's expectations, given the restricted restorative space availability. An interdisciplinary approach involving Endodontic, Periodontic and Prosthodontic treatment procedures. After confirming from the patient about the complexity of treatment, technique sensitivity, cost factor and time required for completion of the procedure; full mouth rehabilitation involving maxillary and mandibular arch was planned. Different philosophies are available for full mouth rehabilitation; Pankey Mann philosophy is the most accepted and practical philosophies for occlusal rehabilitation. It is well organized logical procedure that 


\section{International Journal of Science and Research (IJSR) \\ ISSN (Online): 2319-7064}

Index Copernicus Value (2015): 78.96 | Impact Factor (2015): 6.391

progresses smoothly with less wear and tear on the patient operator and technique. According to Pankey Mann philosophy, treatment is divided into 4 stages.Step 1: Examination, diagnosis, treatment planning and prognosis. Step 2: Harmonization of anterior guidance for the best possible esthetics, function and comfort. Step 3: Selection of acceptable occlusal plane and restoration of lower posterior occlusion in harmony with anterior guidance i.e. in a manner that will not interfere with condylar guidance. Step 4: Restoration of upper posterior occlusion in harmony with anterior guidance and condylar guidance. Based on clinical findings, restricted restorative space availability following treatment procedures were planned. (a) Increasing the VDO by occlusal splint therapy for about4-6 weeks for adaptation of masticatory muscle and TMJ. (b) Surgical crownlengthening procedures $(31,32,41 \& 42)$ and intentional root canal therapy of mandibular anterior teeth (wrt 31, 32 33, 41, 42 \&43), Post \& Core wrt 11. (c) Extraction of hopeless teeth (wrt: $13 \& 14)$.

Examination: A 57-year-old woman was referred to the department of prosthodontics crown and bridge for the treatment of her severely worn dentition. Her chief complaint was that she could not eat anything/ smile fully because her teeth were worn too much. Intra-oral examination revealed a generalized loss of dental substance. The anterior and posterior teeth had sharp enamel edges, dentinal craters, and attritional wear (Fig No: 1)

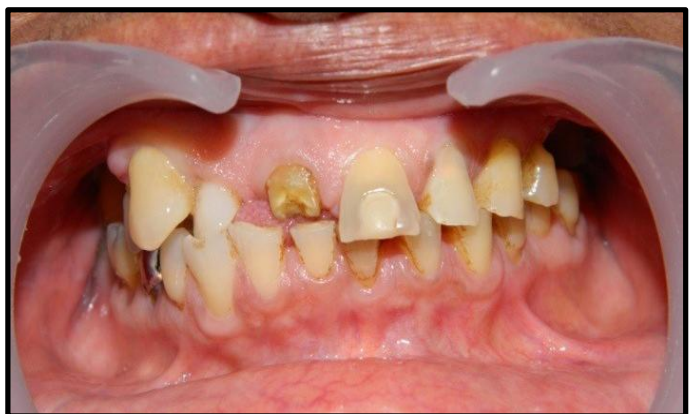

Figure 1: Maxillary And Mandibular Arch Intra Oral View

The management of the worn mandibular anterior dentition is a restorative challenge, and often presents in association with reduced OVD, which further complicates the rehabilitation procedures. The patient did not have any history of Temporo-mandibular disorder and soreness of the mastication muscles, but the discrepancy between centric occlusion (CO) and maximum inter cuspal position (MIP) was found when she was guided to centric relation (CR) with Dawson's bimanual manipulation technique ( Fig No: 2).

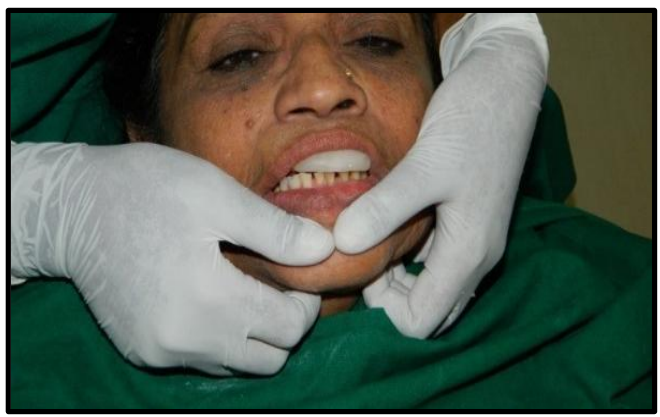

Figure 2: Recording Centric Relation by Dawson's Bimanual Manupilation.
Occlusal Splint therapy: An occlusal bite splint was fabricated and delivered with anterior and posterior teeth coverage, to test an increased vertical dimension of approximately $4 \mathrm{~mm}$. The patient was told that she should wear the splint as much as possible both daytime and night time. The splint was designed to offer bilateral contacts of all posterior teeth in centric relation and guides of the anterior teeth in excursive movement. According to a systematic review investigating the implications of increasing the VDO, a permanent increase in the vertical dimension from 1 to $5 \mathrm{~mm}$ is a safe and reliable procedure, and the associated signs and symptoms are self-limiting with a tendency to resolve within 2 weeks. Although it may have been safe to increase the VDO by as much as $5 \mathrm{~mm}$, in this study, we performed the work carefully, delivering the occlusal bite splint before commencing restorative treatment, and observing for 1 month to confirm adaptation by the patient. The patient was recalled for every week for clinical examination. She reported no Temporo-mandibular joint discomfort, a little muscle tenderness resolved during the first week. The VDO was determined by techniques including assessment of facial appearance and phonetic evaluation [8].

The Maxillary and mandibular diagnostic casts were mounted on a semi-adjustable articulator (Hanau ${ }^{\mathrm{TM}}$ Modular Articulator; Whip Mix Corp., Louisville, USA) using a facebow record and an interocclusal record that was made with the aid of anterior deprogramming device and polyvinylsiloxaneocclusal registration material (EXABITE II; GC Corp., Tokyo, Japan) (Fig No 3). Because the patient's interocclusal rest space was 2 - $3 \mathrm{~mm}$ larger on the premolar area than normal distance, the actual increase were determined $3 \mathrm{~mm}$ in the anterior teeth and $1-2 \mathrm{~mm}$ in the posterior teeth.Anterior guidance was established. In this case, lower anteriors were restored and temporary crowns were given. Esthetics, function and comfort of the patient were assessed.Then came the third part of the treatment i.e. the occlusal plane analysis. We analyzed it using Broadrick flag analyzer. (Fig. No: 4 \& 5). The caliper was set at a radius of 4 inch from needle point to pencil point. Needle point of the caliper is placed against the selected point on premolar and an arc was scribed on the flag (anterior survey line). Caliper point is held against the condyle ball of the articulator and another arc is made in flag (condylar survey line). From the intersect point a line is drawn from molar to canine.

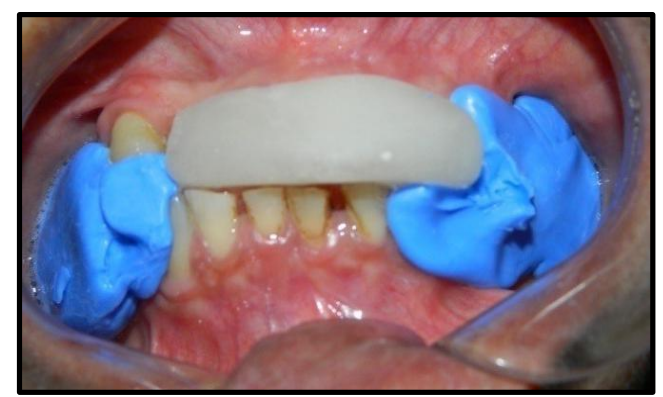

Figure 3: Centric Relation Record With Anterior Deprograming Device. 


\section{International Journal of Science and Research (IJSR) \\ ISSN (Online): 2319-7064}

Index Copernicus Value (2015): 78.96 | Impact Factor (2015): 6.391

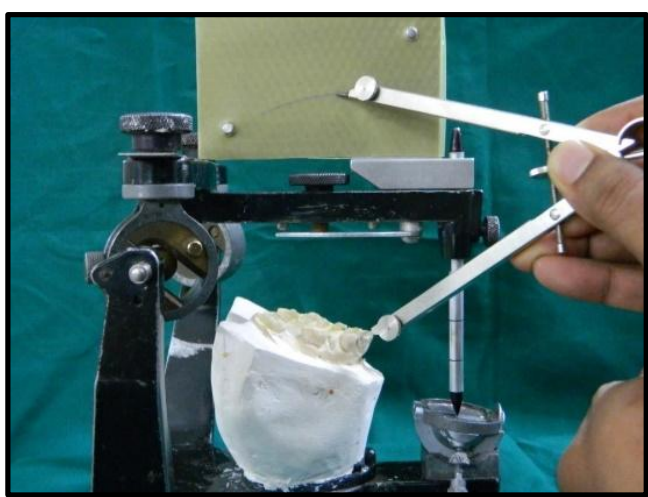

Figure 4: Broadricks Plane Analysis Anterior Survey Line

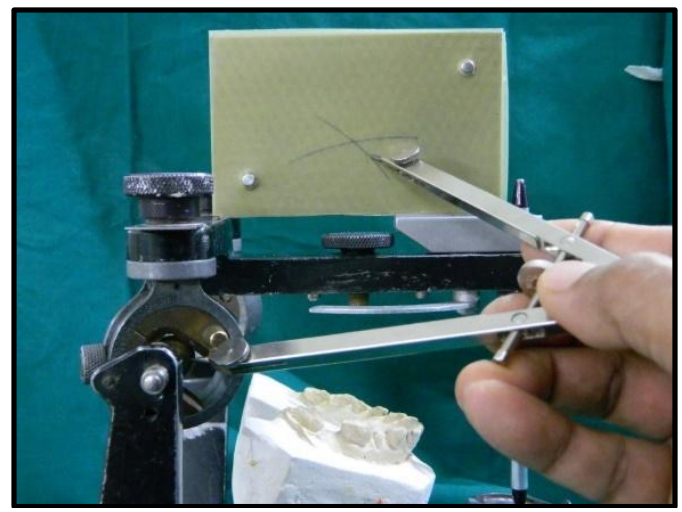

Figure 5: Broadricks Plane Analysis Posterior Survey Line

Thus we got an accepted occlusal plane for the lower posteriors. To transfer this to mouth a wax guide is made and held against the teeth and preparation line $1.5 \mathrm{~mm}$ below the plane is marked on the teeth, giving space for the restoration. Temporization period for two month and patient is reviewed once in two weeks. Patient is comfortable with new bite and permanent crowns and maxillary cast partial denture was fabricated after final cementation of maxillary and mandibular teeth (Fig. No: $6,7 \& 8$ ).

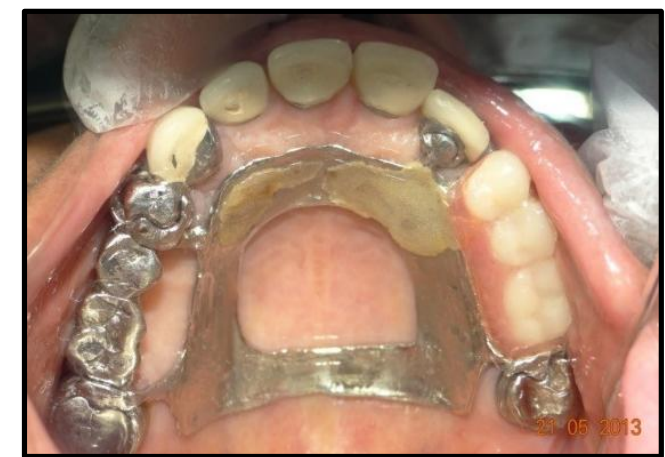

Figure 6: Final Fixed Restoration With Cast Partial Denture For Maxillary Arch.

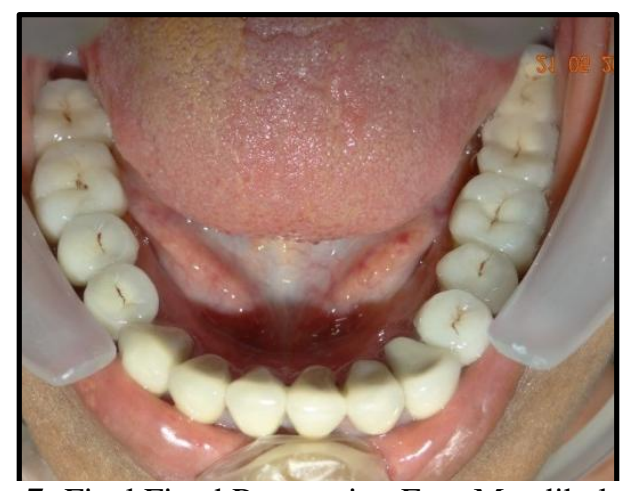

Figure 7: Final Fixed Restoration Foer Mandibular Arch

\section{Discussion}

Oral health, function, esthetics, and comfort are very important in decision making for the treatment of patients for full mouth rehabilitation.In treating such patients a planned and systematic approach is required so that a favorable treatment outcome can result. The possible causes of patient $\mathrm{s}$ worn dentition that might include posterior interferences, para-function, eating habit, and dental ignorance.

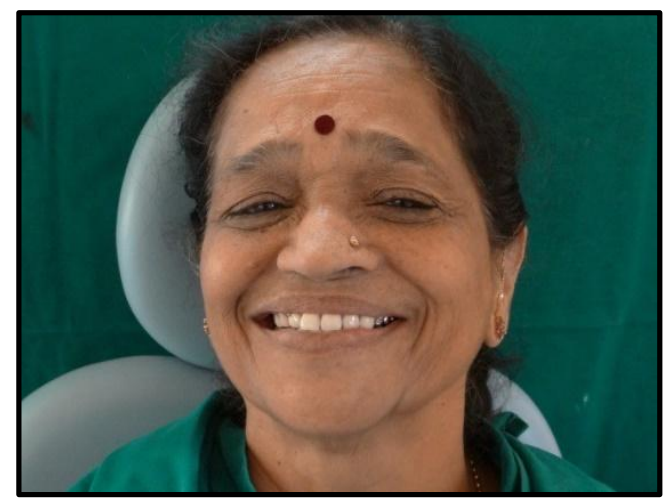

Figure 8: Extra Oral View

Aspects which contributes difficulties associated with tooth wear management include: (i) Challenges associated with deriving an accurate diagnosis. (ii)Uncertainties in knowing at what precise stage to implement active restorative intervention (as opposed to simple passive management and monitoring). (iii) A lack of understanding on how to restore such severely worn dentitions, with the aim of ultimately attaining a functionally and aesthetically stable restored dentition. (iv)A Lack of knowledge relating to the availability of contemporary materials and their respective techniques of application [9].

There are several reported procedures for determination of the VDO, and one commonly employed method is measurement of the free-way space when the mandible is at rest. Nis-wongerreported that the freeway space was $4 / 32$ " ( $3 \mathrm{~mm}$ ) in $87 \%$ of patients; the remaining $13 \%$ varied from $1 / 32$ " to $11 / 32$ ". Nis-wonger concluded that as the teeth slowly wear down, the body adapts by making necessary changes in bone and so tissue to maintain the space. The loss of teeth or wear are potential factors for changes the dynamic nature of the stomatognathic system [10]. 


\section{International Journal of Science and Research (IJSR) \\ ISSN (Online): 2319-7064}

Index Copernicus Value (2015): 78.96 | Impact Factor (2015): 6.391

Various methods to assess change in VDO as follows: (a) Loss of posterior support:mandibular posterior teeth were missing, and the patient did not use the mandibular partial denture. Posterior collapse resulted in excessive wear and fracture of anterior teeth. (b) History of wear: Physiologic wear can be compensated by tooth eruption in general, but the accelerated wear may exceed the rate of eruption. (c) Phonetic evaluation:If the distance between the incisal edge of the mandibular incisors and lingual surface of the maxillary incisors is about $1 \mathrm{~mm}$, it makes normal /s/ sound. The patient's increased space altered /s/ sound to $/ \mathrm{J} /$. (d) Intero-cclusal rest space:The patient's inter-occlusal rest space that was measured between nose tip and chin tip will be $5-6 \mathrm{~mm}$ that is greater than the normal value, $2-4 \mathrm{~mm}$. (e) Facial appearance:Wrinkles and drooping commissures around mouth were observed [11].

Careful assessment of the patient's diet, eating habits and/or gastric disorders, along with the present state of occlusion is essential for appropriate treatment planning. Occlusal splint therapy has been shown to be useful for the diagnosis and management of various masticatory system disorders. Occlusal splints of varied designs and application have been employed in the treatment of myofacial pain dysfunction (MPD) and temporomandibular joint disorders (TMD). These appliances provide the practitioners with a noninvasive, reversible form of intervention to manage the patient's symptoms. An occlusal device should be stable, comfortable and have a proper occlusion so that the patient can use it consistently to assess the effects of occlusal change. Occlusal splint therapy is well advocated for following purposes: (i) To provide diagnostic information, (ii) To protect the teeth, cheek and /or tongue in patients with Bruxism, (iii) To stabilize unstable occlusion, (iv) To promote jaw muscle relaxation in patients with stress related pain symptoms like tension headache and neck pain of muscular origin[12].

Various classifications have been proposed to classify patients requiring full mouth rehabilitation, however, the classification most widely adopted is the one given by Turner and Missirlian in 1984. According to them, patients with occlusal wear can be broadly classified as follows:

- Category-1: Excessive wear with loss of vertical dimension of occlusion (VDO). The patient closest speaking space is more than $1 \mathrm{~mm}$ and the inter-occlusal space is more than $4 \mathrm{~mm}$ and has some loss of facial contour and drooping of the corners of the mouth.

- Category-2: Excessive wear without loss of VDO but with space available. Patients typically have a long history of gradual wear caused by bruxism, oral habits, or environmental factors but the occlusal vertical dimension (OVD) is maintained by continuous eruption.

- Category-3: Excessive wear without loss of VDO but with limited space. There is excessive wear of anterior teeth over a long period, and there is minimal wear of the posterior teeth. Centric relation and centric occlusion are coincidental with a closest speaking space of $1 \mathrm{~mm}$ and an inter-occlusal distance of 2-3 mm. In such cases vertical space must be obtained for restorative materials. This can be accomplished by orthodontic movement, restorative repositioning, surgical repositioning of segments, and programmed OVD modification [13].
There are many philosophies to follow for an occlusal rehabilitation, most important among them is Hobo's philosophy and Pankey Mann Schuyler philosophy. Pankey Mann Schylur philosophy is one of the most practical philosophies for occlusal rehabilitation. It is well organized logical procedure that progresses smoothly with less wear and tear on the patient operator and technique.Optimum oral health should be prime objective of the rehabilitation procedures, because the ultimate goal will always be to restore the mouth to health and preserve this status throughout life of a patient [14]. There has been a search for the ideal occlusal scheme to be followed during full mouth rehabilitation that would provide optimal muscle and joint function besides aiming at restoring the occlusal surfaces of teeth. Many concepts and techniques have been discussed till now in order to rehabilitate dentition with fixed prosthodontics.

On literature review it was found that occlusal schemes were also formulated for oral rehabilitation in patients with periodontal diseases. Youdelis in 1971 proposed an occlusal scheme for advanced periodontitis cases. According to Nyman and Lindhe Scheme for extremely advanced periodontitis cases even contact should be provided in the inter-cuspal position, although no great emphasis is placed upon the type of contacts [12]. Finally, it is dependent on the clinician to choose an appropriate occlusal scheme for a particular reconstruction case after comprehensively reviewing the existing clinical condition so as to achieve predictable long term results and a functional occlusion.

\section{Conclusion}

The treatment of patient with attrition and decreased VDO is of increasing interest. The objective of full mouth rehabilitation must be the reconstruction, restoration, and maintenance of the health of the entire oral mechanism. Pankey Mann Schuyler philosophy the treatment can be completed more quickly and easily and with much more comfort for the patient. With better treatment planning and patient's psychological counseling, dentists can improve patient's quality of life.

Full mouth rehabilitation is a radical procedure and should be carried out in accordance with the dentist's choice of treatment based on his knowledge of various philosophies followed and clinical skills. A comprehensive study and practical approach must be directed towards reconstruction, restoration and maintenance of the health of the entire oral mechanism.

\section{References}

[1] Schuyler C (1939). Problems associated with opening the bite which would contraindicate it as a common procedure. J Am Dent Assoc. 26: 734-40.

[2] Tench R (1938). Dangers in reconstruction involving increase of the vertical dimension of the lower third of the human face. J Am Dent Assoc. 25: 566-70.

[3] Lambrechts P, Braeme M, Vuylsteke-Wauters M, Vanherle G (1989). Quantitative in vivo wear of human enamel. J Dent Res. 68: 1752-1754. 


\section{International Journal of Science and Research (IJSR) \\ ISSN (Online): 2319-7064 \\ Index Copernicus Value (2015): 78.96 | Impact Factor (2015): 6.391}

[4] Eccles J (1982). Tooth surface loss from abrasion, attrition and erosion. Dent Update. 9: 373-381.

[5] A. J. R. Crothers (1992). "Tooth wear and facial morphology," Journal of Dentistry.; 20(6): 333-341,

[6] Dawson PE (2008). Functional occlusion- From TMJ to smile design. $1{ }^{\text {st }}$ edition Newyork; Elsvierinc. 430-452

[7] Somayeh Zeighami,HakimehSiadat,and Sakineh Nikzad (2015). Full Mouth Reconstruction of a Bruxer with Severely Worn Dentition: A Clinical Report.Case Reports in Dentistry. $3: 1-7$

[8] Min-Chieh Hsieh, Chih-Ling Chang ,Wei-Te Victor Lee (2013). Full mouth rehabilitation in a limited restorative space with severely worn teeth - a case report. Journal of Prosthodontics and Implantology. 2(1): 13-17

[9] Mehta, S B; Banerji, S Millar, B J Suarez-feito (2012). Current concepts on the management of tooth wear: part 1. Assessment, treatment planning and strategies for the prevention and the passive management of tooth wear British Dental Journal. 212(1): 17-27.

[10] Niswonger ME (1934). The rest position of the mandible and centric relation. J Am Dent Assoc. 21: 1572-82.

[11] J Abduo, K Lyons (2012). Clinical considerations for increasing occlusal vertical dimension: a review Australian Dental Journal. 57: 2-10

[12] Shweta Choudhary, H Murali Rao, Ajit Kumar Rohilla, CheranjeeviJayam (2015). The Occlusal Splint Therapy: A Literature Review. Indian Journal of Dental Sciences. 7(1): 101-108.

[13] Bhawana Tiwari • KomalLadha • AarutiLalit ${ }^{\bullet}$ B. DwarakanandaNaik (2014). Occlusal Concepts in Full Mouth Rehabilitation: An Overview J Indian Prosthodont Soc. 14(4):344-351.

[14] Okeson JP. (2003) Occlusal Appliance Therapy. In: Management of Temporomandibular Disorders and Occlusion, $5^{\text {th }}$ Ed Mosby Company, St. Louis, Missouri, 509-512

[15] RajulVivek, T.P. Chaturvedi, Atul Bhavnagar et al (2014). Full Mouth Rehabilitation of a Patient with Severely Worn Dentition - A Systematic Clinical Approach.Journal of Dental and Medical Sciences. 13(7): 105-110.

[16] Turner KA, Missirlian DM (1984) Restoration of the extremely worn dentition. J Prosthet Dent. 52:467-474

[17] A. V. Sreekumar, Mohammed Sajeer P. C, Levin Chengappa (2016).A Multidisciplinary Approach to Restoration of Severely Worn Dentition : A Case Report. Journal of Research in Dentistry. 4(2): 63-66

[18] Mi-Young Song, Ji-Man Park, Eun-Jin Park ( 2010). Full mouth rehabilitation of the patient with severely worn dentition: a case report JAdvProsthodont. 2:10610

[19] Jinsa P. Devassy, AnkithaSivadas, ShabasMuhammed (2017).Full mouth occlusal rehabilitation; by Pankey Mann Schuyler philosophy Annals of Prosthodontics \&Restorative Dentistry. 3(1):29-33

[20] MeenakshiKhandelwal,VivekSharma, VikasPunia,SandhyaPunia (2011).Rehabilitation for partially edentulous patient with anteriorly worn dentition. International Journal of Dental Clinics. 3(2):91-92.
[21] D. R. Bloomand J. N. Padayachy (2006).Increasing occlusal vertical dimension - Why, when and how. British Dental Journal. 2(5): 251-256.

[22] P Rajesh, Maruthi Prasad, Sindhu Halal (2014). Full Mouth Rehabilitation of a Patient with AmelogenesisImperfecta: A Case Report Journal of International Oral Health. 6(4):76-79.

[23] Ramesh.P.Nayakar (2011). Esthetic rehabilitation of severely worn dentition: a functional and prospective approach. International Journal of Dental Clinics.3(4):54-55.

\section{Author Profile}

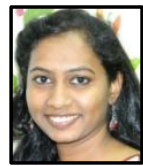

Dr. Shilpa Sinnurkar is Assistant Professor, Department of Prosthodontics Coorg Institute of Dental Sciences, Virajpet -571218 Karnataka India. 